\title{
Space-Based Optical Observations on Space Debris via Multipoint of View
}

\author{
Zhao Li $(\mathbb{D}$, Yidi Wang $\mathbb{D}$, and Wei Zheng \\ College of Aerospace Science and Engineering, National University of Defense Technology, Changsha 410073, China \\ Correspondence should be addressed to Yidi Wang; wangyidi_nav@163.com
}

Received 3 June 2019; Revised 27 January 2020; Accepted 31 January 2020; Published 22 February 2020

Academic Editor: Zhiguang Song

Copyright $\odot 2020$ Zhao Li et al. This is an open access article distributed under the Creative Commons Attribution License, which permits unrestricted use, distribution, and reproduction in any medium, provided the original work is properly cited.

\begin{abstract}
In this paper, the space debris catalogue in LEO is fulfilled using multipoint of view (MPOV) observations constructed by a spacecraft formation. The dual-point of view (DPOV) observation is employed because it has higher cost-benefit ratio than other MPOV methods, and it required that the target is detectable for multiple optical sensors simultaneously. This condition is matched by designing the orbit of formation and the installation angles of sensors without frequent attitude maneuvers. The design parameters are optimized to maximize the matching degree of observation constraints to improve the coverage performance of the formation, and the equivalent ranging error is taken into account to ensure the orbit determination accuracy. Finally, the system capabilities of different schemes are compared by simulations. For the schemes with the same number of platforms, simulation results show that the initial orbit determination accuracy of the proposed scheme is higher.
\end{abstract}

\section{Introduction}

The number of space debris increases since the beginning of human space activities. For space debris larger than $10 \mathrm{~cm}$ in diameter, the number monitored increased from 9949 in 2006 to 160914 in 2010. Currently, the Low Earth Orbit (LEO) and the Geosynchronous Earth Orbit (GEO) environments have become much cluttered because of the huge amounts of space debris [1]. Specifically, the density of debris in sun-synchronous region is the highest. More importantly, the collisions could cause a chain reaction known as the Kessler Syndrome phenomenon, which would destroy all operating satellites in these particular orbital regions [2].

Space debris poses significant risks to the implementation of current and future space missions. Therefore, the active debris removal is necessary to solve this problem [3]. Researchers have proposed different methods for the debris removal [4], and the accurate kinematic status of the target is required in all these methods. So, the space situation awareness (SSA) system is built to catalog the space debris and provide orbital information of the targets for debris removal. Moreover, the SSA system is essential because it can protect the normal operation of spacecraft from being damaged by the debris.

The SSA system can use two kinds of measurement approaches: ground-based measurement and space-based one. Almost all the observations for space debris are currently made by ground-based optical and radar systems. The on-board sensors will be one of the fundamental assets of the future SSA system, because they can significantly improve the system performance [5]. The space-based optical sensor is suitable for SSA system considering its potential of continuous observations, weather independency, and low power consumption. Some technology demonstration satellites have been launched, i.e., the Midcourse Space Experiment (MSX) satellite with the Space-Based Visible (SBV) sensor was launched in 1996 into an $898 \mathrm{~km}$ near sunsynchronous orbit [6], and the SBV sensor was transitioned to a contributing sensor in the space surveillance network. In addition, the European Space Agency (ESA) proposed the space-based optical program [7], in which the observation concept, sensor architecture, and system performance were investigated.

A challenging problem is how to use single space-based optical sensor to fulfil accurate orbit determination of targets. 
The observation arcs are very short, usually only a few minutes, because of the limited field of view (FOV) of optical sensor and high relative angular velocities between observation platform and the space objects. There are many estimation algorithms that have been proposed for the orbit determination from a short-arc angle-only observation, such as genetic algorithms [8], batch [9], or sequential estimators [10-12]. However, in these algorithms, an efficient initial orbit determination (IOD) method is required to guarantee the final convergence, which is still not well solved.

The accurate orbit determination issue can be well solved by using multipoint of view (MPOV) observations in a distributed space-based optical sensor network $[13,14]$. MPOV observation requires that the same object is simultaneously detectable for multiple optical sensors. It can effectively improve the orbit determination accuracy of targets because the observability of tracking system is enhanced. In previous studies, this observation method is implemented by operating the sensors in tracking mode. When the object is detected first by one sensor, the attitude maneuvers will be performed by other sensors to track this object [15]. In this mode, frequent attitude maneuvers are required, which are difficult to implement and inefficient because the relative angular velocities between observation platform and the space objects is about $2 \mathrm{deg} / \mathrm{s}$. These problems can be avoided in scan mode in which the sensor is fixed on platform, and an object will be imaged when it passes through the sensor's FOV and is detectable [16].

The aim of this paper is to propose a new method to catalog the space debris in LEO using a formation composed by multiple spacecraft platforms with optical sensors fixed on. Firstly, dual-point of view (DPOV) observation is chosen because it has higher cost-benefit ratio compared with other MPOV methods. Then, DPOV observation is implemented by designing the orbit of formation and the installation angles of sensors rather than frequent attitude maneuvers. The formation scheme is optimized when the matching degree of observation constraints is taken as the optimization index and the equivalent ranging error is considered. In which, the distance from the target to the observation platform can be calculated by using DPOV observation based on binocular vision. Simulation results show that the mean IOD accuracy for the targets of proposed formation scheme is higher than that of other scheme with the same number of platforms.

The remaining part of the paper is organized as follows: the space-based optical system model is introduced in Section 2. In Section 3, the process of formation design is presented. In Section 4, the system performance is evaluated by simulations to verify the feasibility of proposed scheme. Finally, the main conclusions are summarized in Section 5.

\section{Space-Based Optical System Model}

2.1. Dynamics Model. Earth-centered inertial (ECI) coordinate system is selected, and the orbital dynamics model of space object in LEO is given by [17]:

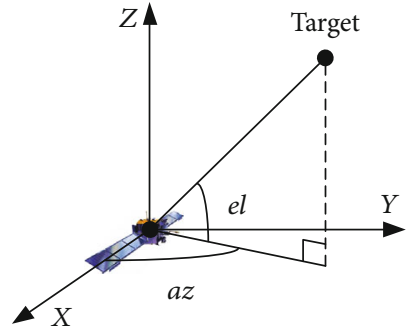

Figure 1: The diagram of optical sensor measurement.

$$
\begin{aligned}
{\left[\begin{array}{c}
\dot{\mathbf{r}} \\
\dot{\mathbf{v}}
\end{array}\right] } & =\left[\begin{array}{l}
\mathbf{v} \\
\mathbf{a}
\end{array}\right], \\
\mathbf{a} & =\mathbf{a}_{T}+\mathbf{a}_{J_{2}}+\mathbf{v}, \\
\mathbf{a}_{T} & =-\mu_{e} \frac{\mathbf{r}}{r^{3}}, \\
\mathbf{a}_{J_{2}} & =\frac{3}{2} J_{2}\left(\frac{R_{e}}{r}\right)^{2}\left(\frac{\mu_{e}}{r^{3}}\right)\left[\begin{array}{l}
x\left(5 \frac{z}{r^{2}}-1\right) \\
y\left(5 \frac{z}{r^{2}}-1\right) \\
z\left(5 \frac{z}{r^{2}}-3\right)
\end{array}\right],
\end{aligned}
$$

in which $\mathbf{r}$ and $\mathbf{v}$ are the position and velocity of the target, $\mathbf{r}=[x, y, z]^{\mathrm{T}}$ and $r=\sqrt{x^{2}+y^{2}+z^{2}} ; \mathbf{a}$ is the acceleration of the target, $\mathbf{a}_{T}$ is the two-body central gravitational acceleration, $\mathbf{a}_{J_{2}}$ is the $J_{2}$ nonspherical perturbation, and $\mathbf{v}=$ $\left[v_{x}, v_{y}, v_{z}\right]^{\mathrm{T}}$ is the process noise; $J_{2} \approx 0.00108263 ; \mu_{e}$ is the Earth gravitational constant and $R_{e}$ is the Earth radius. The influences of other perturbations could be ignored because the observation duration is very short.

2.2. Measurement Model. The detection of debris using optical sensor is done as follows: the passage of an object across the field of view of the sensor during a timeframe results in an image of a streak across a pattern of fixed stars and diffuse background. The right ascension and declination of the target are calculated according to the information of the background stars in the image. As shown in Figure 1, the angle position of the target with respect to the observation platform is expressed by two angular measurements-azimuth and elevation [6]. The measurement models are as follows:

$$
\left\{\begin{array}{l}
a z=\tan ^{-1}\left(\frac{y-y_{j}}{x-x_{j}}\right)+\varepsilon_{1}, \\
e l=\tan ^{-1}\left(\frac{z-z_{j}}{\sqrt{\left(x-x_{j}\right)^{2}+\left(y-y_{j}\right)^{2}}}\right)+\varepsilon_{2},
\end{array}\right.
$$

where $\left[x_{j}, y_{j}, z_{j}\right]^{\mathrm{T}}$ is the position of the $j$ th sensor in ECI coordinates, $j=1, \cdots, N_{\mathrm{sn}}$ and $N_{\mathrm{sn}}$ is the number of sensors, and $\boldsymbol{\varepsilon}=\left[\varepsilon_{1}, \varepsilon_{2}\right]^{\mathrm{T}}$ is the measurement noise. 


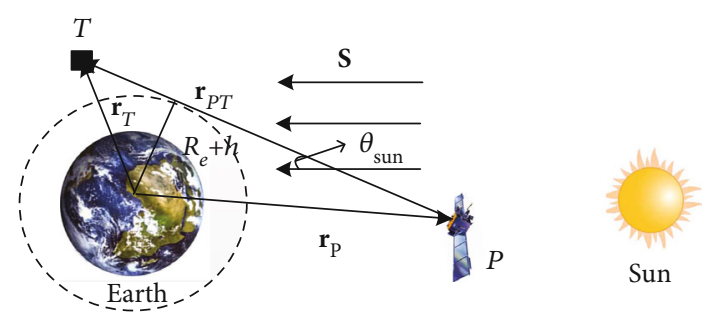

Figure 2: The constraint of Earth occultation and sun illumination.

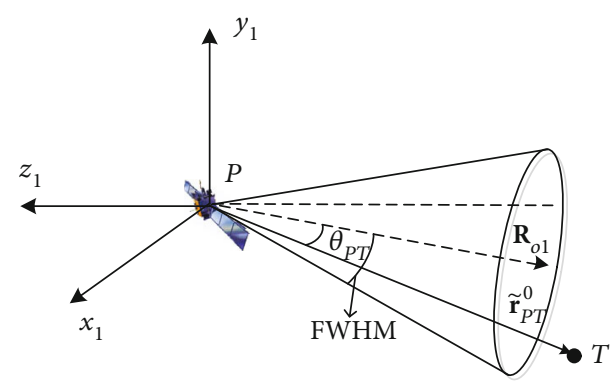

FIGURE 3: The constraint of field of view.

2.3. Observation Constraints. The observation constraints of SBO sensor include the Earth occultation, the sun illumination, the Field of View (FOV), and the target magnitude.

As illustrated in Figure 2, $T$ denotes the target, $P$ denotes the observation platform; $\mathbf{r}_{T}, \mathbf{r}_{P}$ are the position vectors of the target and the observation platform in the Earth-center inertial (ECI) coordinate system, respectively; $\mathbf{S}$ is the sun direction, $R_{e}$ is the Earth radius, and $h$ is the thickness of the Earth atmosphere.

The Earth occultation means that the target is invisible when the connection between the observation platform and the target crosses earth or atmosphere. As shown in Figure 2, this constraint can be expressed as follows:

$$
\begin{aligned}
\left|\mathbf{r}_{P T}\right| & >\sqrt{\left|\mathbf{r}_{P}\right|^{2}-\left(R_{E}+h\right)^{2}}+\sqrt{\left|\mathbf{r}_{T}\right|^{2}-\left(R_{E}+h\right)^{2}}, \\
\mathbf{r}_{P T} & =\mathbf{r}_{T}-\mathbf{r}_{P} .
\end{aligned}
$$

The optical sensors cannot work when it is oriented against the sun. So, the sun illumination constraint is given by

$$
\mathbf{r}_{P T} \cdot \mathbf{S} \geq 0
$$

The FOV of optical sensor is limited and it is the most impact factor to the visibility of space-based surveillance system. Supposing the shape of FOV is circular, as shown in Figure 3, we can obtain this constraint in the local vertical/local horizontal (LVLH) coordinate system of platform according to

$$
\begin{aligned}
& \theta_{P T}=\cos ^{-1}\left(\tilde{\mathbf{r}}_{P T}^{0} \cdot \mathbf{R}_{o 1}\right)<\text { FWHM, } \\
& \tilde{\mathbf{r}}_{P T}^{0}=\frac{\tilde{\mathbf{r}}_{P T}}{\left|\tilde{\mathbf{r}}_{P T}\right|},
\end{aligned}
$$

$$
\tilde{\mathbf{r}}_{P T}=\mathbf{r}_{P T} M_{\mathrm{ECI}}^{\mathrm{LVLH}},
$$

where the positive $x_{1}$-axis points radially outward, the $y_{1}$-axis lies on the fundamental plane and is in the direction of motion on platform orbit, and the $z_{1}$-axis completes the setup to yield a Cartesian dextral system; $\mathbf{R}_{o 1}$ is the optical axis unit vector of sensor in LVLH coordinates, and FWHM is the Full Width Half Maximum of sensor in degree; $\tilde{\mathbf{r}}_{P T}$ is obtained from $\mathbf{r}_{P T}$ by a coordinate system conversion shown as Equation (7).

The constraint of target magnitude means that the target is too dark to be observed. So, the target is detectable when its magnitude is less than the maximal detectable magnitude of sensor. The target magnitude is determined by its albedo, cross-sectional area, and sun-illumination condition, and it is calculated by [18].

$$
\begin{aligned}
m= & -26.7-2.5 \times \log _{10}(\xi \times A)+5 \log _{10}\left|\mathbf{r}_{P T}\right| \\
& +\Delta m\left(\theta_{\text {sun }}\right)<m_{\max }, \\
\Delta m\left(\theta_{\text {sun }}\right)= & -2.5 \lg \left[\sin \theta_{\text {sun }}+\left(\pi-\theta_{\text {sun }}\right) \cos \theta_{\text {sun }}\right],
\end{aligned}
$$

in which $\xi$ is the target albedo, $A$ is the cross-sectional area of the target in the motion direction, and $m_{\max }$ is the maximal detectable magnitude.

2.4. Estimation Algorithm. In this section, the extended information filter (EIF) used for orbit determination of the space targets is introduced [19-22], and its superiority in this mission compared with the extended Kalman filter (EKF) is discussed. Defining the state variable as $\mathbf{x}=\left[x, y, z, v_{x}, v_{y}, v_{z}\right]^{\mathrm{T}}$, the measurement of $j$ th sensor is $\mathbf{z}_{j}=\left[a z_{j}, e l_{j}\right]^{\mathrm{T}}$. So, the time-discrete model of this nonlinear system can be summarized as follows:

$$
\begin{aligned}
\mathbf{x}_{k} & =\boldsymbol{\Phi}_{k, k-1} \mathbf{x}_{k-1}+\mathbf{v}_{k-1}, \\
\mathbf{z}_{k, j} & =\mathbf{H}_{k, j} \mathbf{x}_{k}+\boldsymbol{\varepsilon}_{k, j},
\end{aligned}
$$

where $\boldsymbol{v}_{k-1}$ and $\boldsymbol{\varepsilon}_{k, j}$ are independent zero mean white Gaussian noise with covariance $\mathbf{Q}_{k-1}$ and $\mathbf{R}_{k, j}$, respectively. $\boldsymbol{\Phi}_{k, k-1}$ and $\mathbf{H}_{k, j}$ are the state transition matrix and measurement matrix, and their calculation procedures are given in [15].

The system state $\mathbf{x}$ and covariance matrix $\mathbf{P}$ at the time $k-1$ are known. In the information filter, the information state and information matrix at time $k-1$ are defined by $\mathbf{y}_{k-1}=\mathbf{P}_{k-1}^{-1} \mathbf{x}_{k-1}$ and $\mathbf{Y}_{k-1}=\mathbf{P}_{k-1}^{-1}$, respectively.

In time update step, the predicted information state and information matrix are calculated by

$$
\begin{aligned}
\mathbf{y}_{k / k-1} & =\mathbf{P}_{k / k-1}^{-1} \mathbf{x}_{k / k-1}, \\
\mathbf{Y}_{k / k-1} & =\mathbf{P}_{k / k-1}^{-1}, \\
\mathbf{x}_{k / k-1} & =\boldsymbol{\Phi}_{k, k-1} \mathbf{x}_{k-1}, \\
\mathbf{P}_{k / k-1} & =\boldsymbol{\Phi}_{k, k-1} \mathbf{P}_{k-1} \boldsymbol{\Phi}_{k, k-1}^{\mathrm{T}}+\mathbf{Q}_{k-1} .
\end{aligned}
$$




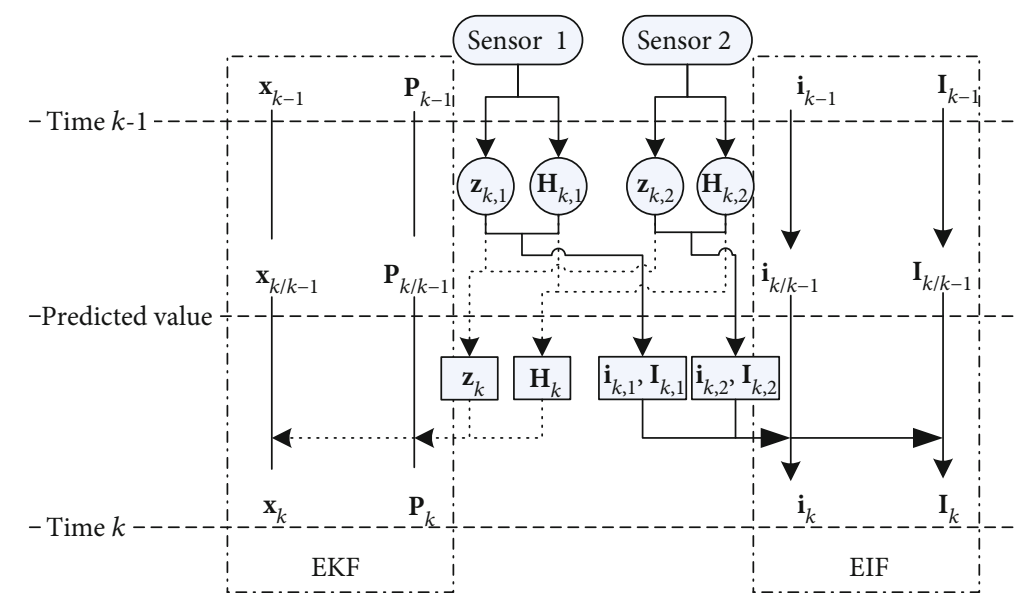

FIgUre 4: Comparison of EKF and EIF.

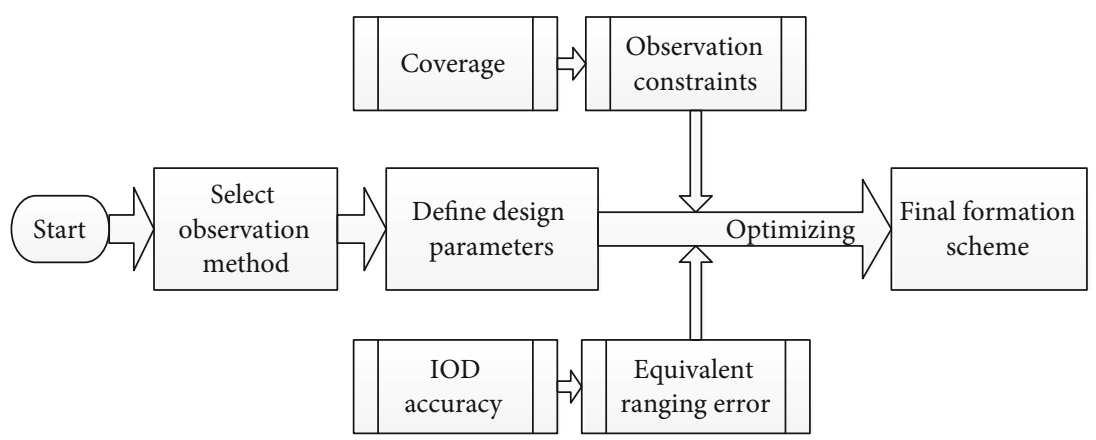

FIGURE 5: Flowchart of formation design.

In measurement update step, the information state and information matrix are updated by

$$
\begin{aligned}
& \mathbf{y}_{k}=\mathbf{y}_{k / k-1}+\sum_{j=1}^{N_{\mathrm{sn}}} \mathbf{i}_{k, j}, \\
& \mathbf{Y}_{k}=\mathbf{Y}_{k / k-1}+\sum_{j=1}^{N_{\mathrm{sn}}} \mathbf{I}_{k, j},
\end{aligned}
$$

where $\mathbf{i}_{k, j}$ and $\mathbf{I}_{k, j}$ are, respectively, the information state contribution and the information matrix contribution, which are as follows:

$$
\begin{aligned}
\mathbf{i}_{k, j} & =\mathbf{H}_{k, j}^{\mathrm{T}} \mathbf{R}_{k, j}^{-1}\left[\left(\mathbf{z}_{k, j}-\mathbf{h}_{j}\left(\mathbf{x}_{k / k-1}\right)\right)+\mathbf{H}_{k, j} \mathbf{x}_{k / k-1}\right], \\
\mathbf{I}_{k, j} & =\mathbf{H}_{k, j}^{\mathrm{T}} \mathbf{R}_{k, j}^{-1} \mathbf{H}_{k, j} .
\end{aligned}
$$

Supposing that the number of SBO sensors is two, the implementation procedures of EKF and EIF are compared in Figure 4.

Based on the above figure, the measurements and measurement matrixes need to be transmitted between sensors in EKF while the information state contribution and the information matrix contribution in EIF. The information state contribution and the information matrix contribution of each sensor are obtained through fusing its measurement and measurement matrix. This means that the messages transmitted in EIF are preprocessed, and the message transmissions in EIF occur later than in EKF. So, the computing burden of each platform is well balanced in EIF because the data processing is accomplished on one of the platforms after message transmission. In addition, the update step is achieved by simply summing the information contributions in EIF, while higher-order matrix is required in EKF.

\section{Formation Design}

As shown in Figure 5, the procedure of the formation design can be roughly divided into three steps. First, the preferable observation method is selected from different kinds of MPOV. Second, the definition of design parameters is given based on the implementation of MPOV observations. Finally, the formation scheme is obtained by optimizing the design parameters when the coverage performance and the IOD accuracy are both considered.

3.1. Analysis of Multipoint of View. Although multipoint of view (MPOV) is used for debris observation in many studies, there is no previous study which has compared the differences in different kinds of MPOV. In this section, the superior observation method is picked out by comparing the 
TABLE 1: Initial conditions of parameters in the simulation.

\begin{tabular}{|c|c|c|}
\hline $\begin{array}{l}\text { (1) Initial condition of the orbit } \\
\text { determination process }\end{array}$ & $\begin{array}{l}\text { Initial state error } \\
\text { Process noise matrix } \\
\text { Filter }\end{array}$ & $\begin{array}{c}\Delta \mathbf{x}_{0}=[10 \mathrm{~km} ; 10 \mathrm{~km} ; 10 \mathrm{~km} ; 10 \mathrm{~m} / \mathrm{s} ; 10 \mathrm{~m} / \mathrm{s} ; 10 \mathrm{~m} / \mathrm{s}] \\
\mathbf{Q}=\operatorname{diag}\left(\left[q_{1}^{2}, q_{1}^{2}, q_{1}^{2}, q_{2}^{2}, q_{2}^{2}, q_{2}^{2}\right]\right), \text { where } q_{1} \text { is } 1 e-2, q_{2} \text { is } 1 e-5 \\
\text { Extended information filter }(\mathrm{EIF})\end{array}$ \\
\hline \multirow{4}{*}{ (2) Initial condition of the optical sensor } & Measurement period & $1 \mathrm{~s}$ \\
\hline & Measurement error & $5 \operatorname{arcsec}$ \\
\hline & FWHM & $10 \mathrm{deg}$ \\
\hline & $\begin{array}{l}\text { Maximal detectable } \\
\text { magnitude }\left(m_{\max }\right)\end{array}$ & 18 \\
\hline \multirow{2}{*}{ (3) Initial condition of the targets } & Albedo $(\xi)$ & 0.3 \\
\hline & Cross-sectional area $(A)$ & $100 \mathrm{~cm}^{2}$ \\
\hline
\end{tabular}

TABLE 2: The initial orbital elements of observation platforms.

\begin{tabular}{lcccccc}
\hline Observation method & No. of platform & $a(\mathrm{~km})$ & $e$ & $i(\mathrm{deg})$ & $\Omega(\mathrm{deg})$ & $u(\mathrm{deg})$ \\
\hline \multirow{2}{*}{ Dual-point of view $(1,2)$} & 1 & 7178 & 0 & 98.6 & 300 & 303 \\
Three-point of view $(1,2,3)$ & 2 & 7178 & 0 & 98.6 & -5 \\
& 3 & 7178 & 0 & 98.6 & 297 \\
\hline
\end{tabular}

TABLE 3: The initial orbital elements of targets.

\begin{tabular}{lcccccc}
\hline No. of target & $a(\mathrm{~km})$ & $e$ & $i(\mathrm{deg})$ & $\Omega(\mathrm{deg})$ & $\omega(\mathrm{deg})$ & $f(\mathrm{deg})$ \\
\hline 1 & 7177 & $1 e-6$ & 85.4 & 136.6 & 159.5 & 0 \\
2 & 7268 & $1 e-6$ & 89.6 & 336.9 & 0 & 306.5 \\
3 & 7525 & $1 e-6$ & 72.2 & 0 & 350.3 \\
\hline
\end{tabular}

performance of dual-point of view (DPOV) and three-point of view (TPOV). The initial conditions of parameters that would be used in the simulation are listed in Table 1. This simulation focuses on the orbit determination performance of different observation methods under the same observation arc. Supposing MPOV observations are realized through attitude maneuvers, and the observation duration is set as $500 \mathrm{~s}$. The initial orbital elements of observation platforms and targets are given in Table 2 and Table 3. The orbital elements include semimajor $(a)$, eccentricity $(e)$, orbital inclination $(i)$, RAAN $(\Omega)$, argument of perigee $(\omega)$, true anomaly $(f)$, and arguments of latitude $(u, u=\omega+f)$.

Figure 6 presents the averages of position and velocity errors obtained by 100 Monte Carlo runs. As we can see, the convergence rate of TPOV is slightly faster than that of DPOV, and there are no significant differences in the steady accuracy between two observation methods. This means that the improvement of target tracking capacity is not remarkable when the number of observation platform increases. Moreover, due to more constraints need to be match, the observation arc length of TPOV is usually shorter than that of DPOV when the constraint of FOV is considered. This may weaken the performance of TPOV. In conclusion, DPOV is more suitable for the space surveillance system because it has higher cost-benefit ratio.
3.2. Optimizing Formation Scheme. In this section, DPOV observations on the space targets are realized with sensors in scan mode. The formation scheme is optimized when the coverage performance and the orbit determination accuracy are considered.

3.2.1. The Design Parameters. To ensure a favourable sunillumination condition during the entire mission, the dawndusk, sun-synchronous orbit is suitable for the observation on debris because it can ensure a full use of the optical sensors [11]. As shown in Figure $7,(\cdot)_{1}$ and $(\cdot)_{2}$ are values of $(\cdot)$ for platforms 1 and 2.

Two platforms are distributed in two sun-synchronous orbits with the same orbit altitude. The relationship between orbit altitude and orbit inclination is expressed by the following equation [23]:

$$
-\frac{3}{2} \frac{\sqrt{\mu_{e}} J_{2} R_{e}^{2}}{\left(1-e^{2}\right)^{2}\left(R_{e}+H\right)^{7 / 2}} \cos i=\dot{\Omega}_{\text {sun }} \text {, }
$$

where $H$ is the orbit altitude and $\dot{\Omega}_{\text {sun }}$ is the sun rotation rate with respect to the ECI coordinates. Supposing that the orbits are circular and the absolute phase of satellites can hardly influence the system performance. Therefore, the formation 

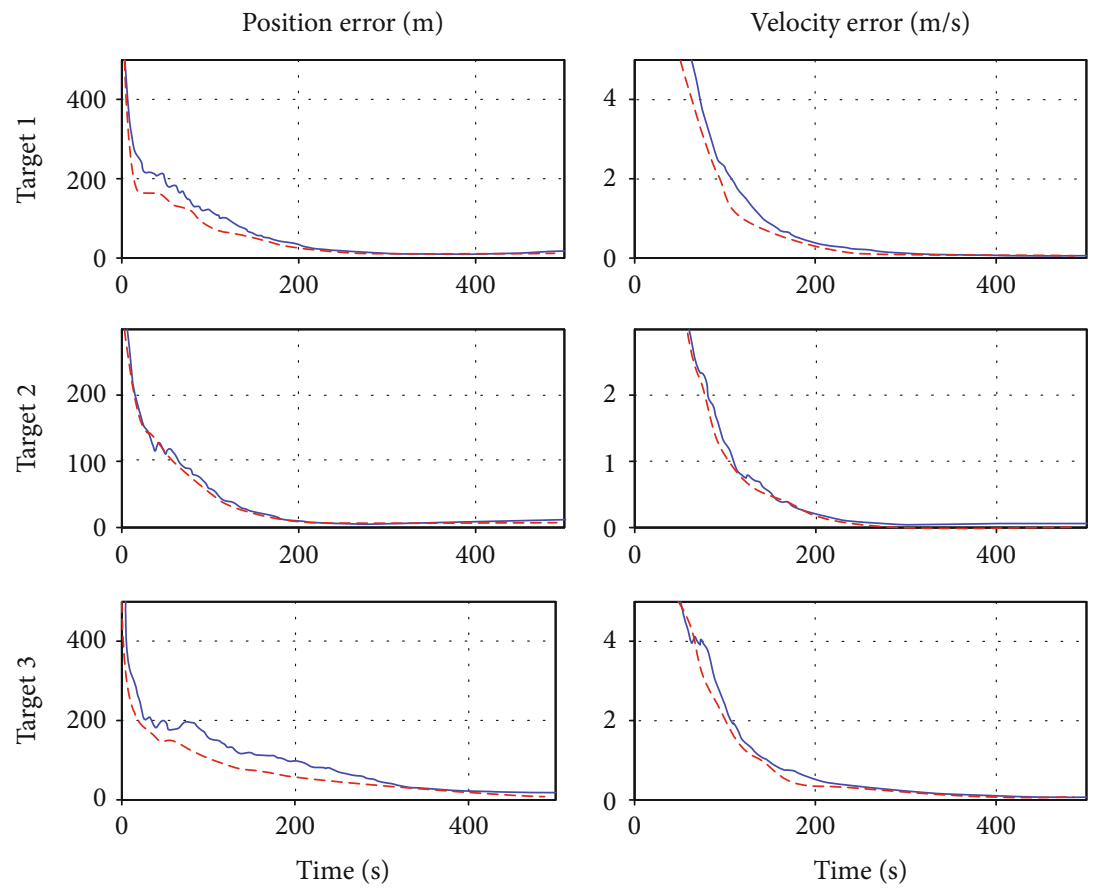

- DPOV
-- TPOV

FIGURE 6: Position and velocity errors of targets 1, 2, and 3 for different observation methods.

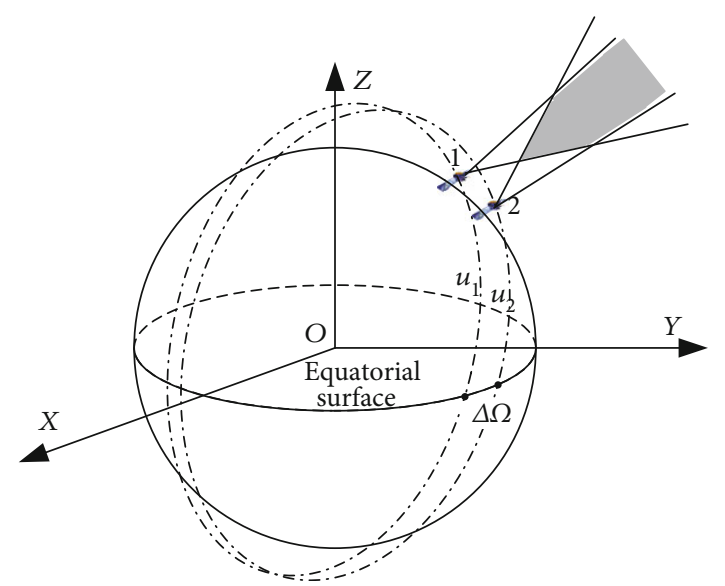

FIGURE 7: Formation involving two sun-synchronous orbits.

configuration is determined by the orbit altitude $(H)$, the RAAN difference $(\Delta \Omega)$, and the phase difference $(\Delta u)$ between two platforms.

The optical sensors are fixed on the platforms, and their installation angles are designed to make the field of view of the two sensors overlap in space to achieve the dual-point of view observations. Supposing the body and the LVLH coordinates of each platform coincide because the threeaxis stable attitude control system is applied. To simplify the description, the sensor pointing is defined in LVLH coordinates. As illustrated in Figure 8, the LVLH coordinate system is defined by the unit vectors $\mathbf{x}_{1}, \mathbf{y}_{1}$, and $\mathbf{z}_{1}, \mathbf{R}_{o 1}$ is the optical axis unit vector of sensor, and $\mathbf{R}_{o x z}$ is the projection of $\mathbf{R}_{o 1}$ on $x o z$-plane.

In the above figure, the installation angles are expressed by $\alpha$ and $\beta$. In which, $\alpha$ is the included angle between $\mathbf{R}_{o y z}$ and $\mathbf{R}_{o 1}$, and $\beta$ is the included angle between $\mathbf{R}_{o x z}$ and the negative $z_{1}$-axis. $\mathbf{R}_{o 1}$ is expressed by

$$
\mathbf{R}_{o 1}=\cos \alpha \sin \beta \mathbf{x}_{1}-\sin \alpha \mathbf{y}_{1}-\cos \alpha \cos \beta \mathbf{z}_{1} .
$$

In order to simplify the formation design, supposing the relationships between the installation angles of two platforms are given by

$$
\begin{aligned}
& \alpha_{1}=-\alpha_{2}=\alpha, \\
& \beta_{1}=\beta_{2}=\beta .
\end{aligned}
$$

Thus, the design parameters of the formation include $H$, $\Delta \Omega, \Delta u, \alpha$, and $\beta$.

3.2.2. Constraint of the Equivalent Ranging Error. In the previous study [11], the coverage performance is the only factor considered in formation design. However, the IOD accuracy of targets is also a crucial indicator of system capability. So, the equivalent ranging error is considered as a constraint condition to ensure the IOD accuracy in the formation design, and its definition and calculation process are as follows.

Supposing that the target is located at the intersection of the optical axes of the two sensors, and the simplified 


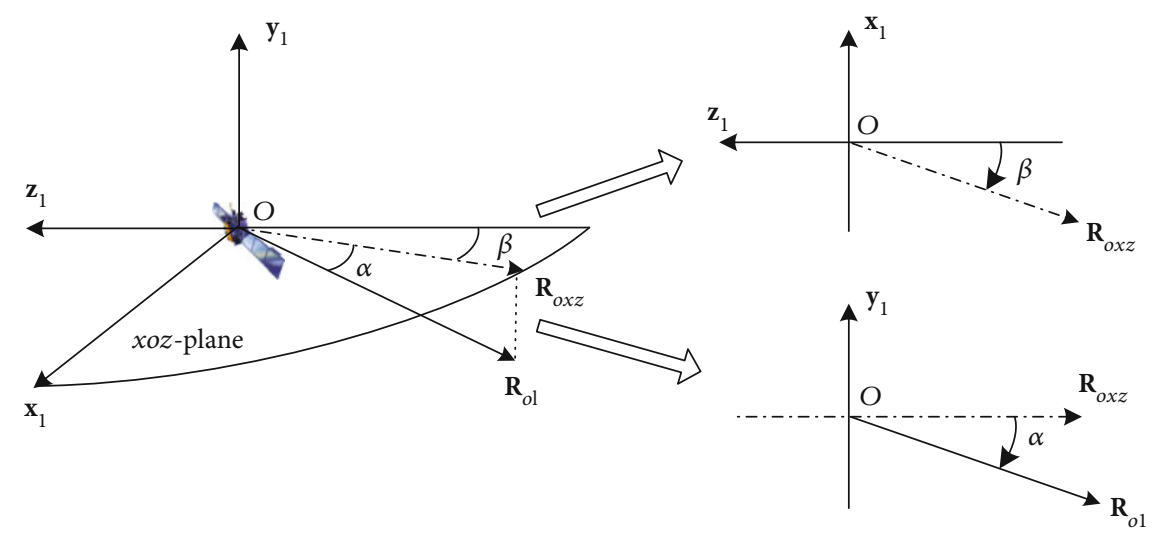

FIGURE 8: The installation angles of optical sensor.

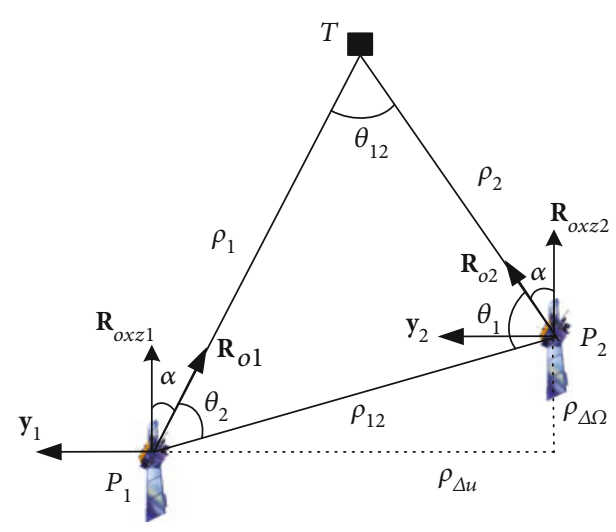

FIGURE 9: The geometry of dual-point of view observation.

geometry of dual-point of view observation is shown in Figure 9.

In the above figure, $\mathbf{R}_{o 1}$ and $\mathbf{R}_{o 2}$ are the optical axes unit vector of sensors 1 and 2 , respectively; $\rho_{1}$ is the distance between platform 1 and the target, $\rho_{2}$ is the distance between platform 2 and the target, $\rho_{12}$ is the distance between platform 1 and platform 2 , and $\rho_{12}=\sqrt{\rho_{\Delta u}^{2}+\rho_{\Delta \Omega}^{2}} ; \rho_{\Delta u}$ and $\rho_{\Delta \Omega}$ can be obtained by

$$
\begin{gathered}
\rho_{\Delta u} \approx 2 \pi\left(R_{e}+H\right) \frac{\Delta u}{360}, \\
\rho_{\Delta \Omega} \approx 2 \pi\left(R_{e}+H\right) \frac{\Delta \Omega}{360} .
\end{gathered}
$$

According to sine theorem, the distance between the target and the platform can be calculated by

$$
\rho_{1}=\frac{\sin \theta_{1}}{\sin \theta_{12}} \rho_{12}
$$

The equivalent ranging error $\Delta \rho_{1}$ is that the distance error caused by angle measurement error of sensor, and it can be obtained by

$$
\Delta \rho_{1}=\left|\tilde{\rho}_{1}-\rho_{1}\right|=\left|\left(\frac{\sin \left(\theta_{1}+\Delta \theta\right)}{\sin \theta_{12}}-\frac{\sin \theta_{1}}{\sin \theta_{12}}\right)\right| \rho_{12},
$$

in which $\tilde{\rho}_{1}$ is the range between platform 1 and the target when the measurement error is considered, and the $\Delta \theta$ is the angle measurement error.

As we have known from Figure 9, there are

$$
\begin{aligned}
& \theta_{1}=\pi-\alpha-\tan ^{-1}\left(\frac{\Delta u}{\Delta \Omega}\right), \\
& \theta_{12}=\cos ^{-1}\left(\mathbf{R}_{o 1} \cdot \mathbf{R}_{o 2}\right) .
\end{aligned}
$$

According to Equations (14) and (15), there is

$$
\mathbf{R}_{o 2}=\cos \alpha \sin \beta \mathbf{x}_{2}+\sin \alpha \mathbf{y}_{2}-\cos \alpha \cos \beta \mathbf{z}_{2} .
$$

Substituting Equations (14) and (22) into Equation (21), supposing the corresponding axes of the LVLH coordinates for two platforms are parallel to each other, so

$$
\theta_{12}=\cos ^{-1}\left(\cos ^{2} \alpha-\sin ^{2} \alpha\right)=2 \alpha \text {. }
$$

Substituting Equations (20) and (23) into Equation (19), there is

$\Delta \rho_{1}=\left|\left(\frac{\sin \left(\alpha+\tan ^{-1}(\Delta u / \Delta \Omega)+\Delta \theta\right)}{\sin (2 \alpha)}-\frac{\sin \left(\alpha+\tan ^{-1}(\Delta u / \Delta \Omega)\right)}{\sin (2 \alpha)}\right)\right| \rho_{12}$.

In order to ensure the IOD accuracy of targets, it is supposed that $\Delta \rho_{1}$ should be less than $1 \mathrm{~km}$.

3.2.3. Analysis of Optimization Results. The space debris in LEO region are mainly concentrated near the sunsynchronous orbit, especially in $600-900 \mathrm{~km}$ altitudes with inclinations about 80-100 degrees. Due to this region with serious pollution has high economic value, it is selected as the surveillance object of proposed formation. Specifically, a target set including 100 debris has been selected based on the orbit distribution of space objects in this region, and its 
TABLE 4: The initial distribution of the debris population (100 objects).

\begin{tabular}{lccccc}
\hline$a(\mathrm{~km})$ & $e$ & $i(\mathrm{deg})$ & $\Omega(\mathrm{deg})$ & $\omega(\mathrm{deg})$ & $f(\mathrm{deg})$ \\
\hline Normal $\left(7178,100^{2}\right)$ & $1 e-6$ & Normal $\left(80,10^{2}\right)$ & Uniform $(0,360)$ & 0 & Uniform $(0,360)$ \\
\hline
\end{tabular}
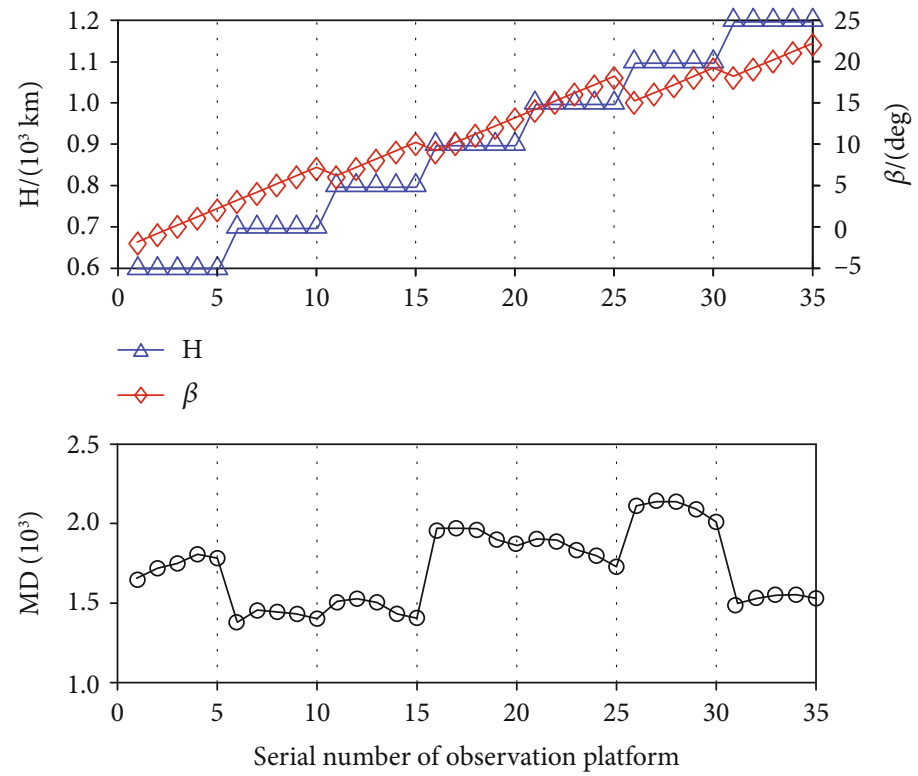

Figure 10: The matching degrees of single platforms with different $H$ and $\beta$.

initial distribution is reported in Table 4. In which, "Normal $\left(\mu, \sigma^{2}\right)$ " means the Normal distribution with mean $\mu$ and variance $\sigma^{2}$, and "Uniform $[\mathrm{a}, \mathrm{b}]$ " means the Uniform distribution in the interval from a to $b$.

The matching degree (MD) of observation constraints is defined to indicate the coverage performance, that is, the total number of discrete points that matches all observation constraints simultaneously. It can be calculated by

$$
\begin{aligned}
\mathrm{MD} & =\sum_{i=1}^{K} \lambda_{i}, \\
\lambda_{i} & = \begin{cases}1, & \text { all observation constraints matched } \\
0, & \text { else }\end{cases}
\end{aligned}
$$

where $K$ is the total number of simulation steps.

Then, the optimization problem for formation design is formulated as follows:

$$
\left\{\begin{array}{lc}
\max & \mathrm{MD} \\
\text { s.t. } & \Delta \rho_{1}<1 \mathrm{~km} .
\end{array}\right.
$$

In order to reduce the search space of design parameters, the optimization process is divided into two steps. Firstly, the appropriate values of $H$ and $\beta$ are determined by analysing the matching degrees of single platform. Then, the matching degree of formation with two platforms under different $\Delta u$,
$\Delta \Omega$, and $\alpha$ is researched to obtain the preferable values for the rest design parameters. The simulation duration is $24 \mathrm{~h}$, the sampling period is set as $10 \mathrm{~s}$, and the other initial conditions are given in Table 1. The simulation results are as follows.

In Figure 10, the upper subgraph shows the parameter settings for alternative observation platforms with different serial numbers, and the under one demonstrates the corresponding matching degree. Every five alternative platforms with the same orbit altitude is a group. As can be seen in Figure 10 , there is an appropriate value for $\beta$ to make the matching degree reach the peak in each group, and this value increases with the increase of orbit altitude for observation platform. This is because that the orbital altitudes of most targets are lower than those of the platform, and $\beta$ is used to handle the altitude gaps between the platform and targets. The maximum value of matching degree appears when the orbit altitude is $1100 \mathrm{~km}$ and $\beta$ is $16 \mathrm{deg}$. So, this parameter setting is selected for the proposed formation. Based on this, the influences of the rest design parameters on the matching degree are researched. The simulation results are shown in Figure 11 .

In the above figure, the upper subgraph shows the parameter settings for alternative two-spacecraft formations with different serial numbers, and the under one demonstrates the corresponding matching degree. Every five alternative formations with the same $\Delta u$ is a group. As can be seen, the matching degree of formation decreases with the increase of $\Delta u$. There is an appropriate value for $\alpha$ to make the matching 


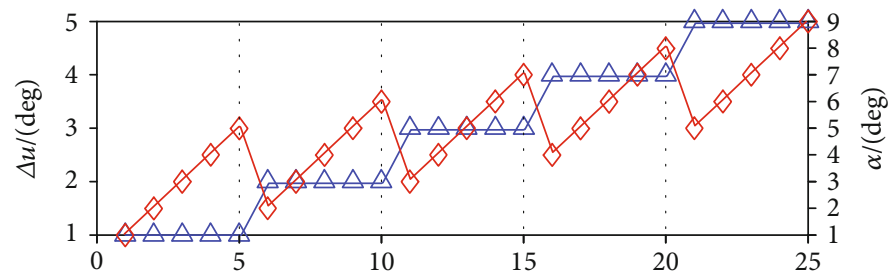

$$
\begin{aligned}
& \diamond \alpha \\
& \triangle \Delta u
\end{aligned}
$$

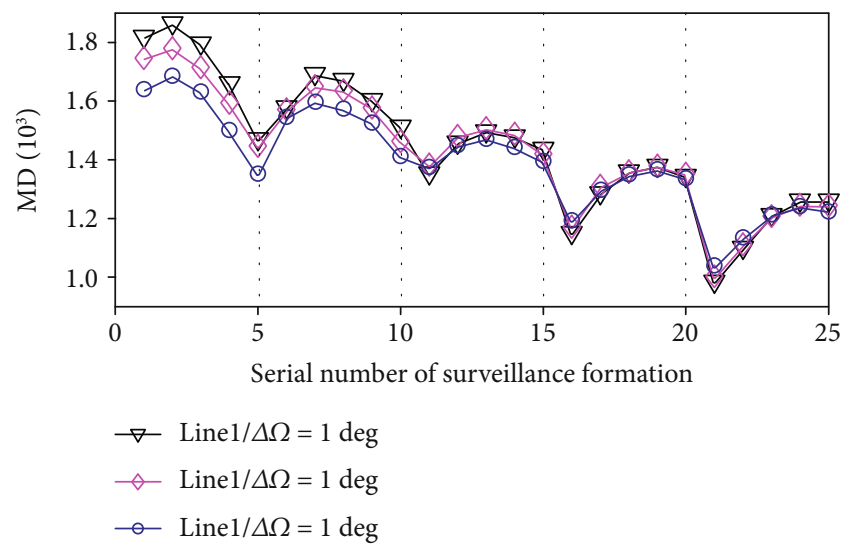

Figure 11: The matching degrees of two-spacecraft formations with different $\Delta u, \Delta \Omega$, and $\alpha$.

TABLE 5: The equivalent ranging errors under different design parameters.

\begin{tabular}{cccccrr}
\hline & $\Delta u(\mathrm{deg})$ & 1 & 2 & 3 & 4 & 5 \\
& $\alpha(\mathrm{deg})$ & 2 & 3 & 5 & 422.6 \\
\hline \multirow{4}{*}{$\Delta \rho(\mathrm{m})$} & $\Delta \Omega=1^{\circ}$ & 1297.0 & 1152.9 & 622.1 & 404.1 \\
& $\Delta \Omega=2^{\circ}$ & 1297.0 & 1152.8 & 621.9 & 422.4 & 403.9 \\
& $\Delta \Omega=3^{\circ}$ & 1296.9 & 1152.7 & 621.8 & 422.2 & 403.6 \\
\hline
\end{tabular}

TABLE 6: The design parameters of proposed formation.

\begin{tabular}{lcccc}
\hline$H(\mathrm{~km})$ & $\beta(\mathrm{deg})$ & $\Delta u(\mathrm{deg})$ & $\Delta \Omega(\mathrm{deg})$ & $\alpha(\mathrm{deg})$ \\
\hline 1100 & 16 & 3 & 2 & 5 \\
\hline
\end{tabular}

degree reach the peak in each group, and this value increases with the increase of $\Delta u$. Moreover, the appropriate value of $\alpha$ is not affected by $\Delta \Omega$ by comparing Line 1 , Line 2 , and Line 3 . This is because the value of $\alpha$ is mainly determined by the along-track distance between two platforms. According to Equation (24), when the angle measurement error of sensor is $5 \mathrm{arcsec}$, the equivalent ranging error under different design parameters can be obtained. The results are given in Table 5.

From the above table, the equivalent ranging error decreases with the increase of $\Delta u$, and $\Delta \Omega$ has no significant effect on the equivalent ranging error. In order to maximize the matching degree while the constraint of equivalent ranging error is met, $\Delta u$ and $\alpha$ should be $3 \mathrm{deg}$ and $5 \mathrm{deg}$, respectively. In this parameter setting, the matching degree
TABLE 7: The initial orbital elements of observation platforms.

\begin{tabular}{lccccc}
\hline Observation platform & $a(\mathrm{~km})$ & $e$ & $i\left(^{\circ}\right)$ & $\Omega\left(^{\circ}\right)$ & $u\left(^{\circ}\right)$ \\
\hline A & 7478 & 0 & 99.94 & 300 & 0 \\
B & 7478 & 0 & 99.94 & 302 & -3 \\
C & 7478 & 0 & 99.94 & 302 & -2 \\
D & 7478 & 0 & 99.94 & 298 & -3 \\
\hline
\end{tabular}

TABLE 8: The formation members and the installation angles for sensors of different schemes.

\begin{tabular}{lccc}
\hline \multirow{2}{*}{ No. of scheme } & Formation members & \multicolumn{3}{c}{ Installation angles $\left(^{\circ}\right)$} \\
\hline 0 & A & 16 & 0 \\
1 & A, B & 16 & $\alpha_{\mathrm{A}}=5, \alpha_{\mathrm{B}}=-5$ \\
2 & $\mathrm{~A}, \mathrm{C}$ & 16 & $\alpha_{\mathrm{A}}=3, \alpha_{\mathrm{C}}=-3$ \\
3 & $\mathrm{~A}, \mathrm{~B}, \mathrm{D}$ & 16 & $\alpha_{\mathrm{A}}=5, \alpha_{B}=\alpha_{D}=-5$ \\
\hline
\end{tabular}


TABLE 9: The coverage performances of different schemes.

\begin{tabular}{|c|c|c|c|c|c|c|c|}
\hline \multirow[t]{2}{*}{ No. of scheme } & \multirow[t]{2}{*}{ Number of platforms } & \multirow[t]{2}{*}{ Coverage } & \multirow[t]{2}{*}{ Number of detected objects } & \multirow[t]{2}{*}{ Mean observation time (s) } & \multicolumn{3}{|c|}{$\begin{array}{l}\text { The distribution of } \\
\text { observation time (s) }\end{array}$} \\
\hline & & & & & $<100$ & $100 \sim 300$ & $>300$ \\
\hline 0 & 1 & SC & 79 & 258 & 22 & 34 & 23 \\
\hline 1 & 2 & CC & 45 & 261 & 14 & 19 & 12 \\
\hline 2 & 2 & DC & 46 & 294 & 13 & 18 & 15 \\
\hline 3 & 3 & TC & 44 & 225 & 16 & 18 & 10 \\
\hline
\end{tabular}

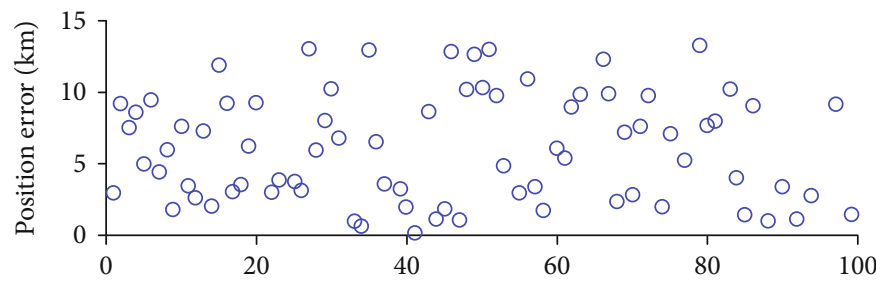

O Scheme 0

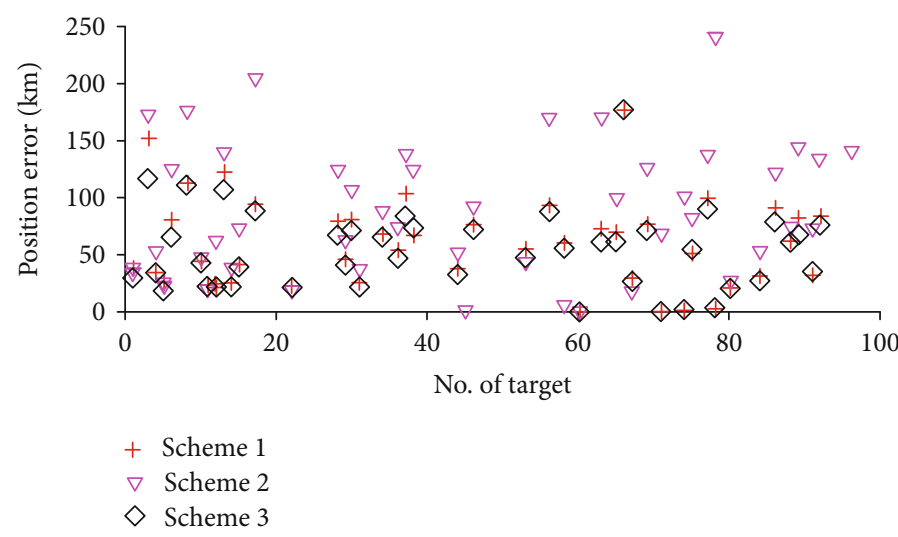

FIgURE 12: Position errors for difference schemes.

at Line 2 is the largest among three lines in Figure 11, so $\Delta \Omega$ should be 2 deg. In summary, the selected values of design parameters are listed in Table 6.

\section{Performance Evaluation}

In this section, we will compare the capabilities of the different schemes to verify the feasibility of proposed scheme in Section 3. The initial conditions of parameters that would be used in the simulation are listed in Table 1, the target set is the same as that using in Section 3.2, the initial orbital elements of platforms are provided in Table 7 , and the formation members and the installation angles for sensors of different schemes are given in Table 8. Among them, the scheme 1 is the proposed one, and the others are used for comparison.

4.1. Coverage on the Targets. Firstly, the coverage performance of different schemes is analysed, including the number of detected objects and mean observation time. The simulation duration is $24 \mathrm{~h}$; the sampling time is set as $1 \mathrm{~s}$.
The simulations focus on the influences of observation methods, so only observation arcs visible to all sensors in the formation are counted. The types of coverage include Single Coverage (SC), Double Coverage (DC), and Three Coverage (TC). And the simulation results are shown in Table 9.

Based on the above table, we can know that the numbers of detected objects of scheme 0 are significantly more than those of other schemes. This is because that MPOV observations need to match more constraints than the single-point of view observations. For formations including two platforms with different design parameters, the mean observation time of scheme 2 is slightly better than that of scheme 1 , which is consistent with the results in the Section 3.2. As we can see, the coverage performance of the proposed formation scheme is not very satisfactory. This problem could be solved by employing periodical attitude maneuver for sensors, which is not considered in this paper.

4.2. Accuracy of Initial Orbit Determination. Next, position errors of the targets for different schemes are compared to 
TABLE 10: The initial orbit determination accuracy of different schemes.

\begin{tabular}{|c|c|c|c|c|c|c|c|}
\hline \multirow[t]{2}{*}{ No. of scheme } & \multirow{2}{*}{$\begin{array}{l}\text { Number of } \\
\text { platforms }\end{array}$} & \multirow{2}{*}{$\begin{array}{c}\text { Number of } \\
\text { detected objects }\end{array}$} & \multirow{2}{*}{$\begin{array}{c}\text { Mean observation } \\
\text { time (s) }\end{array}$} & \multirow{2}{*}{$\begin{array}{l}\text { Mean position } \\
\text { error }(\mathrm{m})\end{array}$} & \multicolumn{3}{|c|}{$\begin{array}{c}\text { The distribution of position } \\
\text { errors (m) }\end{array}$} \\
\hline & & & & & $<10$ & $10 \sim 100$ & $100-300$ \\
\hline 1 & 2 & 45 & 261 & 64.73 & 4 & 21 & 10 \\
\hline 2 & 2 & 46 & 294 & 89.12 & 3 & 25 & 18 \\
\hline 3 & 3 & 44 & 225 & 60.85 & 4 & 34 & 6 \\
\hline
\end{tabular}

evaluate the accuracies of initial orbit determination. The observation arcs used for orbit determination are shown in Table 9. As shown in Figure 12, the results present the average value of position errors over 100 Monte Carlo runs.

As can be seen in the above figure, the position errors of scheme 0 are obviously larger than those of other schemes. This indicates that the accurate IOD can hardly be realize by using single observation platform without attitude tracking. Meanwhile, the position errors of the schemes 1, 2, and 3 are all less than $300 \mathrm{~m}$. This means that the IOD accuracy can be greatly improved by employing MPOV observations. In order to figure out the differences among schemes 1, 2, and 3 , the position errors of objects under different schemes are further analysed in Table 10.

According to the above table, for the formations including two platforms, the mean position errors of scheme 1 are better than those of scheme 2 when its mean observation time is short than that of scheme 2. This indicates that the equivalent ranging error has a greater impact on the orbit determination accuracy than the observation arc length. This conclusion proves that it is necessary to consider the equivalent ranging error in the process of the formation design to ensure the IOD accuracy of targets. And the mean position error of scheme 3 is close to that of scheme 1, which verifies the analysis in Section 3.1. In summary, the scheme 1 is the optimum choice in all schemes demonstrated because it has a good balance between the coverage performance and the orbit determination accuracy.

\section{Conclusions}

In this paper, the multipoint of view observations is proposed for cataloguing the space debris in specific region of LEO. The main conclusions can be summarized as follows: firstly, DPOV is a preferable observation method because it has higher cost-effectiveness ratio than other MPOV methods. Secondly, DPOV can be realized in scan mode by designing the orbit of formation and the installation angles of sensors. This method can greatly reduce the application difficulty of DPOV because the frequent attitude maneuvers in tracking mode are no longer required. Thirdly, the design parameters of proposed formation are optimized to maximize the matching degree when the constraints of the equivalent ranging error are taken into account. The simulation results show that the proposed formation is suitable for the debris catalogue missions. The IOD accuracy of the proposed formation is superior because the equivalent ranging error is taken into account.

\section{Data Availability}

The data used to support the findings of this study are available from the corresponding author upon request.

\section{Conflicts of Interest}

The authors declare that there is no conflict of interest regarding the publication of this paper.

\section{Acknowledgments}

This work was supported by the Foundation of Science and Technology on Space Intelligent Control Laboratory (KGJZDSYS-2018-01) and the National Natural Science Foundation of China (61703413).

\section{References}

[1] H. Schaub, L. E. Z. Jasper, P. V. Anderson, and D. S. McKnight, "Cost and risk assessment for spacecraft operation decisions caused by the space debris environment," Acta Astronautica, vol. 113, pp. 66-79, 2015.

[2] K. Maniwa, T. Hanada, and S. Kawamoto, "Instability of the current space debris population in low earth orbit," Journal of the Japan Society for Aeronautical and Space Sciences, vol. 58 , no. 674, pp. 83-89, 2010.

[3] G. Feng, W. Li, and H. Zhang, "Geomagnetic energy approach to space debris deorbiting in a low Earth orbit," International Journal of Aerospace Engineering, vol. 2019, Article ID 5876861, 18 pages, 2019.

[4] M. Shan, J. Guo, and E. Gill, "Review and comparison of active space debris capturing and removal methods," Progress in Aerospace Sciences, vol. 80, pp. 18-32, 2016.

[5] X. Zhang, J. Xiang, and Y. Zhang, "Space object detection in video satellite images using motion information," International Journal of Aerospace Engineering, vol. 2017, Article ID 1024529, 9 pages, 2017.

[6] G. H. Stokes, C. V. Braun, R. Sridharan, and J. Sharma, "The space-based visible program," Lincoln Laboratory Journal, vol. 11, no. 2, pp. 205-238, 1998.

[7] T. Flohrer, H. Krag, H. Klinkrad, and T. Schildknecht, "Feasibility of performing space surveillance tasks with a proposed space-based optical architecture," Advances in Space Research, vol. 47, no. 6, pp. 1029-1042, 2011.

[8] L. Ansalone and F. Curti, "A genetic algorithm for initial orbit determination from a too short arc optical observation," Advances in Space Research, vol. 52, no. 3, pp. 477-489, 2013.

[9] G. Sciré, F. Santoni, and F. Piergentili, "Analysis of orbit determination for space based optical space surveillance system," Advances in Space Research, vol. 56, no. 3, pp. 421-428, 2015. 
[10] Y. Wang, S. Sun, and L. Li, "Adaptively robust unscented Kalman filter for tracking a maneuvering vehicle," Journal of Guidance Control and Dynamics, vol. 37, no. 5, pp. 1696-1701, 2014.

[11] K. Li, L. Chang, and B. Hu, "A variational Bayesian-based unscented Kalman filter with both adaptivity and robustness," IEEE Sensors Journal, vol. 16, no. 18, pp. 6966-6976, 2016.

[12] Y. Huang, Y. Zhang, Z. Wu, N. Li, and J. Chambers, "A novel adaptive Kalman filter with inaccurate process and measurement noise covariance matrices," IEEE Transactions on Automatic Control, vol. 63, no. 2, pp. 594-601, 2018.

[13] L. Felicetti and M. R. Emami, "A multi-spacecraft formation approach to space debris surveillance," Acta Astronautica, vol. 127, pp. 491-504, 2016.

[14] B. Jia, K. D. Pham, E. Blasch, D. Shen, Z. Wang, and G. Chen, "Cooperative space object tracking using space-based optical sensors via consensus-based filters," IEEE Transactions on Aerospace and Electronic Systems, vol. 52, no. 4, pp. 19081936, 2016.

[15] L. Felicetti and M. R. Emami, "Attitude coordination of multiple spacecraft for space debris surveillance," Advances in Space Research, vol. 59, no. 5, pp. 1270-1288, 2017.

[16] J. Du, J. Chen, B. Li, and J. Sang, "Tentative design of SBSS constellations for LEO debris catalog maintenance," Acta Astronautica, vol. 155, pp. 379-388, 2019.

[17] R. H. Battin, An Introduction to the Mathematics and Methods of Astrodynamics, AIAA, 1999.

[18] W. E. Krag, Visible Magnitude of Typical Satellites in Synchronous Orbits, Massachusetts Institute of Technology, ESD-TR74-278, 1974.

[19] B. Jia, X. Ming, and Y. Cheng, "Multiple sensor estimation using the sparse Gauss-Hermite quadrature information filter," in 2012 American Control Conference (ACC), Montreal, QC, Canada, June 2012.

[20] L. Chang, F. Qin, A. Li, and B. Hu, "Unscented type Kalman filter: limitation and combination," IET Signal Processing, vol. 7, no. 3, pp. 167-176, 2013.

[21] Y. Wang, W. Zheng, S. Sun, and L. Li, "Robust information filter based on maximum correntropy criterion," Journal of Guidance, Control, and Dynamics, vol. 39, no. 5, pp. 11261131, 2016.

[22] D. Simon, Optimal State Estimation: Kalman, H Infinity, and Nonlinear Approaches, John Wiley and Sons, 2006.

[23] D. A. Vallado, Fundamentals of Astrodynamics and Applications, Microcosm Press, El Segundo, CA, USA, 2nd edition, 2001. 